\title{
CARACTERÍSTICAS MORFOLÓGICAS DE FORMAS CULTIVADAS, SILVESTRES E INTERMEDIAS DE FRIJOL COMÚN DE HÁBITO TREPADOR
}

\author{
MORPHOLOGICAL TRAITS OF CULTIVATED, WILD AND WEEDY FORMS IN CLIMBING \\ COMMON BEAN
}

\author{
Rogelio Lépiz Ildefonso*, J. Jesús López Alcocer, J. Jesús Sánchez González, Fernando Santacruz- \\ Ruvalcaba, Ricardo Nuño Romero y Eduardo Rodríguez Guzmán
}

\author{
Profesores Investigadores del Centro Universitario de Ciencias Biológicas y Agropecuarias, Universidad de Guadalajara. Km 15.5 Carretera Guadalajara- \\ Nogales. 45110, Las Agujas, Municipio de Zapopan, Jalisco, México. Tel. (33) 3777-1150.

\section{RESUMEN}

Se hizo una caracterización morfológica de formas cultivadas, silvestres e intermedias de frijol común (Phaseolus vulgaris L., Fabaceae) de hábito trepador. Se evaluaron 15 materiales en un diseño de bloques completos al azar con tres repeticiones. Para determinar las relaciones morfológicas entre las formas incluidas, se registraron 18 variables cuantitativas con lo que se hicieron análisis de varianza, de agrupamiento con base en la distancia euclidiana y componentes principales. Doce de los 18 caracteres registraron diferencias $(P \leq \mathbf{0 . 0 5})$ entre los frijoles domesticados y silvestres, 10 entre domesticados y tipos maleza, $y$ cinco entre intermedios $y$ silvestres. La forma domesticada mostró valores mayores a la silvestre en longitud y anchura de vaina, longitud, anchura y espesor de semilla y peso de 100 semillas; el peso de 100 semillas fue de 5.04 g a 42.93 g. Las variables longitud de tallo y número de entrenudos, no mostraron diferencias entre las formas de frijol trepador. Los caracteres número de vainas por planta y número de semillas por vaina identificados como componentes del rendimiento, no se redujeron en la forma domesticada; estos caracteres se asocian con el mayor potencial de rendimiento de los frijoles cultivados. Dentro de formas de frijol, los genotipos presentaron poca variación morfológica. Las formas domesticadas, silvestres e intermedias de frijol común de hábito trepador, constituyen tres grupos con características morfológicas diferentes, donde los de tipo intermedio forman un grupo morfológicamente más cercano a las poblaciones silvestres.

Palabras clave: Phaseolus vulgaris, domesticación, formas silvestre, cultivada e intermedia.

\section{SUMMARY}

A morphologic characterization of domesticated, wild and weedy forms of climbing common bean (Phaseolus vulgaris L., Fabaceae) was carried out. Fifteen genotypes were evaluated under a randomized complete block design with three replications. In order to know the relationships between included forms, 18 quantitative variables were registered. Data were analyzed by analysis of variance, cluster analysis based on euclidian distance and principal components. Twelve of 18 traits showed differences $(P \leq 0.05)$ between domesticated and wild forms, 10 between domesticated and weedy types, and five between weedy and wild beans. Domesticated forms were higher in length and width of pod, seed length, width and thickness and seed weight, compared to wild forms; the weight of 100 grains varied from 5.04 to $42.93 \mathrm{~g}$. The length of stem and number of nodes were not different among forms. The number of pods per plant and seeds per pod recognized as yield components, did not show reductions in the domesticated form; these traits are associated with high yield potential in cultivated forms. Within each bean form the materials presented little morphological variation. The domesticated, wild and weedy forms of common bean of climber growth habit, constitute three groups with different morphological traits. The weedy form comprises an intermediate group, morphologically closer to wild beans.

Index words: Phaseolus vulgaris, domestication, wild, cultivated and intermediate forms.

\section{INTRODUCCIÓN}

En un periodo de 7000 a 8000 años y con influencia de las fuerzas evolutivas de mutación, migración, deriva genética y selección empírica practicada por el hombre, el frijol común (Phaseolus vulgaris L., Fabaceae) ha transitado desde la forma silvestre de hábito trepador $\mathrm{y}$ semillas pequeñas distribuida en las tierras altas de Mesoamérica y Zona Andina, a la forma actual que se siembra en un amplio rango de ambientes y sistemas de producción (Gepts y Debouck, 1991). El proceso conocido como "síndrome de la domesticación" produjo cambios morfológicos, fisiológicos y genéticos (Koinange et $a l .$, 1996). En los aspectos morfológicos, el hombre centró su atención en semillas de mayor tamaño y de colores más atractivos, vainas más grandes y menos dehiscentes (Debouck e Hidalgo, 1985); adicionalmente, ocurrió la aparición de formas arbustivas y el moteado de la testa de la semilla (Kaplan y Kaplan, 1988). 
Con base en las relaciones entre las formas cultivadas y silvestres determinadas por el uso de marcadores bioquímicos, moleculares y polimorfismo del ADN en los cloroplastos, a la fecha se acepta que la domesticación ocurrió en cada uno de los dos acervos genéticos ancestrales existentes, Mesoamericano (México hasta Colombia) y Andino (Perú hasta el norte de Argentina) (Gepts et al., 1986; Chacón et al., 2005; Kwak y Gepts, 2009). Según Kwak et al. (2009), en Mesoamérica la domesticación del frijol común ocurrió en la cuenca del Río Lerma-Santiago de México. Por otra parte, los tipos de faseolina encontrados en las formas silvestre y cultivada de ambos centros de diversidad, la existencia de arcelina sólo en frijol silvestre y los análisis con marcadores moleculares, evidencian menor variabilidad genética de las formas cultivadas que en los frijoles silvestres, y apoyan la afirmación de un severo efecto fundador en el proceso de domesticación del frijol común (Gepts et al., 1986; Koenig et al., 1990; Kwak y Gepts, 2009).

Los cambios morfológicos más notables ocurridos en la planta de frijol durante el proceso de domesticación, son: de raíz fibrosa fasciculada a fibrosa de diferentes tamaños; de tallo trepador con 20-30 nudos a arbustivo con 5-12 nudos; de entrenudos largos de 10-20 cm a cortos de 2-5 cm; de foliolos pequeños o intermedios a foliolos pequeños a grandes; de inflorescencia lateral a terminal, en los frijoles de hábito determinado; de vainas fuertemente dehiscentes de $6-8 \mathrm{~cm}$ de longitud y $5-8$ óvulos, a dehiscentes o indehiscentes, de $4-30 \mathrm{~cm}$ y $2-9$ óvulos; de semillas pequeñas de 6-14 g en 100 semillas, a pequeñas a grandes de $20-100 \mathrm{~g}$ en 100 semillas (Gepts y Debouck, 1991).

La semilla de las formas silvestres presenta latencia debido a la impermeabilidad temporal de la testa de la semilla, que impide la penetración del agua; en cambio, las semillas de las formas cultivadas germinan pronto después de entrar en contacto con suficiente humedad. Adicionalmente, las formas silvestres reaccionan al fotoperiodo y florecen solamente en fotoperiodos cortos (10$12 \mathrm{~h}$ ), mientras que los materiales domesticados pertenecientes a las razas Mesoamérica y Nueva Granada, son insensibles a la duración del día (Singh et al., 1991; Gepts y Debouck, 1991).

Algunos caracteres morfológicos han permitido comparar la variabilidad genética entre las formas silvestres y cultivadas, tales como: hábito de crecimiento y tipo de semilla (color, tamaño y forma), dos caracteres que son indudablemente más variables entre los frijoles domesticados. Los estudios de cruzamientos han mostrado que los caracteres que separan a los frijoles silvestres y cultivados, son controlados por un reducido número de genes de efectos mayores en el fenotipo, con excepción del tamaño de la semilla (Gepts y Debouck, 1991). Aún para tamaño de semilla, usualmente considerado como carácter poligénico, se ha referido que un locus simple posee un marcado efecto (Koinange et al., 1996). Por lo anterior, se puede afirmar que las diferencias genéticas entre formas silvestre y domesticada de frijol, se deben al efecto de pocos loci (Gepts y Debouck, 1991; Poncet et al., 2004).

La planta del frijol domesticado es anual, herbácea, de hábito arbustivo para siembras en unicultivo o trepador para siembras generalmente asociadas con maíz (Zea mays L.). El ciclo biológico puede variar desde 85 a $270 \mathrm{~d}$, en función del hábito de crecimiento, del clima de la región y de la época de siembra. Aunque se cultiva desde Argentina hasta Canadá y desde el nivel del mar hasta los $3200 \mathrm{~m}$ de altura, es una planta termófila susceptible a heladas (Gepts y Debouck, 1991).

La forma silvestre de $P$. vulgaris es de hábito indeterminado trepador en la que predominan las flores de color morado; las vainas son pequeñas y delgadas, dehiscentes y de granos pequeños; las semillas son generalmente de color gris, ya sea uniformes o jaspeadas. Se le encuentra desde el norte de México hasta el noroeste de Argentina, en una distribución continua y en un rango altitudinal de 500 a $2000 \mathrm{~m}$, con precipitaciones anuales de 500 a $1800 \mathrm{~mm}$. Prospera en ambientes de bosque con baja población de árboles, con preferencia en sitios con arbustos espinosos y maleza; éstos se pueden encontrar en las riberas de los ríos, hábitat con perturbaciones por efectos de vulcanismos, incendios naturales o por modificaciones hechas por el hombre (Toro et al., 1990).

Dentro de la gran diversidad del frijol común, además de los frijoles silvestres y cultivados, existe un grupo intermedio llamado en inglés "weedy form" o "weedy type" frecuentemente denominado como frijol tipo maleza o forma enmalezada, producto de la cruza entre frijoles silvestres y variedades domesticadas. Papa y Gepts (2003), mediante marcadores moleculares, confirmaron que existe flujo genético entre las dos formas principales y que los frijoles intermedios son más cercanos a la forma silvestre. La forma tipo maleza se ha observado en los Andes y también en Mesoamérica (Delgado et al., 1988; Debouck et al., 1993); produce pocas semillas por vaina (4.2 vs. 5.8) pero más grandes (11.9 vs. $6.3 \mathrm{~g}$ en 100 semillas) que la forma silvestre (Delgado et al., 1988; Zizumbo et al., 2005).

Dado que en el Occidente de México existe una gran variabilidad genética del género Phaseolus, que es parte del centro mesoamericano de domesticación del frijol común y que se han colectado 184 muestras de semilla de 
formas silvestres e intermedias de $P$. vulgaris, el objetivo del presente trabajo fue hacer una caracterización morfológica para determinar las relaciones entre las formas cultivadas, silvestres e intermedias de frijol común, y postular los cambios morfológicos ocurridos durante el proceso de domesticación.

\section{MATERIALES Y MÉTODOS}

El trabajo se llevó a cabo en el ciclo primavera-verano 2005, en un campo ubicado en el km. 15.5 de la carretera Guadalajara-Nogales. Se evaluaron 15 poblaciones de frijol común, tres silvestres, dos domesticadas y 10 tipo maleza, todas de hábito indeterminado trepador de la Raza Jalisco (Cuadro 1). En los frijoles silvestres e intermedios se utilizó semilla colectada en 2003 y 2004 en la región Occidente de México; en los cultivados se usó semilla 2004 del proyecto de frijol de la Universidad de Guadalajara. Los genotipos intermedios se identificaron en el campo al momento de su colecta, por presentar mayor longitud de vainas, semillas de mayor tamaño y colores parecidos a los genotipos domesticados.

Se utilizó un diseño de bloques completos al azar con tres repeticiones (una en invernadero y dos en campo), una parcela experimental de dos surcos de $4 \mathrm{~m}$ de largo, $80 \mathrm{~cm}$ entre surcos y una planta cada $20 \mathrm{~cm}$. La semilla de las poblaciones silvestres se escarificó para la siembra. Se fertilizó a la siembra con $40 \mathrm{~N}-40 \mathrm{P}-0 \mathrm{~K}$, la maleza se eliminó manualmente y se instalaron espalderas con estacas, alambre e hilo. Para el registro de la información se tomaron 30 plantas elegidas al azar por parcela y por repetición.

Se registraron 18 variables cuantitativas durante el desarrollo del cultivo y postcosecha (Cuadro 2) y se efectuaron análisis de varianza y comparaciones de medias (Tukey, 0.05) entre formas y materiales. Se aplicó un análisis de agrupamiento con base en la distancia euclidiana con las 18 variables cuantitativas previamente estandarizadas. Estos análisis se llevaron a cabo con el programa NTSYS 2.1 (Rohlf, 2000). Para la inspección de la matriz de las 18 variables cuantitativas, se utilizó la técnica multivariada conocida como Biplot (Sánchez, 1995), que tiene como base la metodología de componentes principales y presenta de manera gráfica las similitudes entre las poblaciones incluidas, las relaciones entre las variables que caracterizan a las poblaciones y los valores relativos de las observaciones para cada vector variable.

\section{RESULTADOS Y DISCUSIÓN}

Hubo diferencias significativas $(\mathrm{P} \leq 0.05)$ entre formas de frijol (Cuadro 2). Al contrastar los valores promedio de las variables por forma de frijol, 12 de los caracteres registraron diferencias $(\mathrm{P} \leq 0.05)$ entre los frijoles domesticados y los silvestres. En longitud de hipocotilo y epicotilo, longitud y anchura de hoja simple, longitud del foliolo central, longitud y anchura de vaina, longitud de ápice, longitud, anchura y espesor de la semilla y peso de 100 semillas, las poblaciones domesticadas mostraron valores superiores a las formas silvestres.

Cuadro 1. Origen y coordenadas geográficas de los sitios de procedencia de las poblaciones de frijol utilizadas en la caracterización morfológica.

\begin{tabular}{|c|c|c|c|c|c|}
\hline Población ${ }^{\dagger}$ & Sitio & Latitud & Longitud & Altitud (m) & Color de semilla \\
\hline ROL 241 (S) & Jala, Nay. & $21^{\circ} 06^{\prime}$ & $104^{\circ} 27^{\prime}$ & 1161 & Gris jaspeado \\
\hline ROL 242 (S) & Magdalena, Jal. & $20^{\circ} 59^{\prime}$ & $104^{\circ} 07^{\prime}$ & 1366 & Gris jaspeado \\
\hline ROL 227-1 (I) & San Pedro, Jal. & $20^{\circ} 49^{\prime}$ & $104^{\circ} 04^{\prime}$ & 1472 & Bayo \\
\hline ROL 227-2 (I) & San Pedro, Jal. & $20^{\circ} 49^{\prime}$ & $104^{\circ} 04^{\prime}$ & 1472 & Negro \\
\hline ROL 227-3 (I) & San Pedro, Jal. & $20^{\circ} 49^{\prime}$ & $104^{\circ} 04^{\prime}$ & 1472 & Café \\
\hline ROL 161 (I) & Tingüindín, Mich. & $19^{\circ} 53^{\prime}$ & $102^{\circ} 32^{\prime}$ & 1859 & Gris pinto \\
\hline ROL 172-1 (I) & Jala, Nay. & $21^{\circ} 08^{\prime}$ & $104^{\circ} 29^{\prime}$ & 1368 & Gris jaspeado \\
\hline ROL 172-2 (I) & Jala, Nay. & $21^{\circ} 08^{\prime}$ & $104^{\circ} 29^{\prime}$ & 1368 & Bayo jaspeado \\
\hline ROL $172-3$ (I) & Jala, Nay. & $21^{\circ} 08^{\prime}$ & $104^{\circ} 29^{\prime}$ & 1368 & Café jaspeado \\
\hline ROL 168 (I) & Zapopan, Jal. & $20^{\circ} 44^{\prime}$ & $103^{\circ} 30^{\prime}$ & 1578 & Café ojo de cabra \\
\hline
\end{tabular}

\footnotetext{
${ }^{\dagger} \mathrm{S}=$ silvestre; $\mathrm{I}=$ intermedio; $\mathrm{D}=$ domesticado.
} 
En longitud de vaina y peso de 100 semillas, los valores fueron de $7.15 v s .11 .96 \mathrm{~cm}$ y de $4.68 v s .42 .93 \mathrm{~g}$, respectivamente; en ambos caracteres el incremento logrado por el proceso de domesticación es evidente y de manera especial en tamaño de semilla, variable que aumentó en 9 veces. Los resultados de esta caracterización muestran que las formas de frijol cultivada y silvestre, son dos grupos morfológicamente diferentes.

Lo anterior concuerda con lo consignado en la literatura, donde se informa del incremento en tamaño logrado en algunos órganos de la planta de frijol por el proceso de domesticación (Gepts y Debouck, 1991); y muy especialmente del tamaño alcanzado en el fruto y semilla (Kaplan y Kaplan, 1988; Gepts y Debouck, 1991). En este sentido, Gepts y Debouck (1991) señalaron que las vainas pueden ser de 6 a $8 \mathrm{~cm}$ y de 4 a $30 \mathrm{~cm}$ de longitud, en la forma silvestre y cultivada, respectivamente y que el peso de 100 semillas puede variar de 6 a $14 \mathrm{~g}$ en los silvestres y de 20 a $100 \mathrm{~g}$ en 100 semillas en los cultivados.

Los caracteres longitud de hipocotilo y epicotilo, longitud y anchura de hoja simple, longitud del foliolo central, longitud y anchura de la vaina, longitud, anchura y espesor de la semilla, variaron en el mismo sentido que el peso de 100 semillas. Estos resultados concuerdan con Gepts y Debouck (1991), quienes asentaron que el tamaño de semilla se correlaciona positivamente con la longitud de las hojas y vainas, y que las mayores dimensiones de estos órganos en la forma domesticada puede deberse a un subproducto de la selección por tamaño de semilla, más que por un proceso conciente de selección.

Aún cuando no se detectaron diferencias significativas para número de vainas por planta y para número de semillas por fruto, la tendencia de menores valores en las poblaciones domesticadas se puede atribuir al efecto de compensación entre los componentes del rendimiento; es decir, al aumentar las dimensiones de vaina y grano en la planta, se reduce el número de frutos y de granos por vaina (Adams, 1967; White e Izquierdo, 1989). En longitud de tallo y número de nudos del tallo tampoco hubo diferencias $(P \geq 0.05)$ entre las formas estudiadas. La igualdad en estos dos caracteres podría significar que durante el proceso de domesticación del frijol trepador, no fue de interés para los humanos cambiar el hábito de crecimiento trepador, probablemente por su mayor competitividad con maleza y arbustos y en las siembras asociadas con maíz.

En la forma intermedia de frijol conocida como tipo maleza, con excepción de longitud de tallo y número de nudos, para todas las variables se registraron valores intermedios entre las formas cultivada y silvestre (Cuadro 2). Al comparar la forma tipo maleza con la forma domesticada, las diferencias fueron significativas ( $\mathrm{P} \leq$ 0.05 ) en 10 de las 18 variables analizadas. En longitud de hipocotilo y epicotilo, longitud y anchura de hoja simple, longitud y anchura de vaina, longitud, anchura y espesor de la semilla y peso de 100 semillas, las poblaciones intermedias mostraron valores menores a los registrados en las poblaciones cultivadas. Este resultado es similar a lo observado entre las formas domesticada y silvestre, e indica también que los frijoles intermedios constituyen un grupo diferente a los frijoles cultivados.

Al contrastar los promedios de las variables en las formas intermedia y silvestre, las diferencias sólo fueron significativas $(\mathrm{P} \leq 0.05)$ en cinco de los 18 caracteres (Cuadro 2). El grupo de los frijoles intermedios fue superior en longitud de epicotilo, longitud y anchura de hoja simple, longitud de la vaina y peso de 100 semillas lo que concuerda con lo observado por Delgado et al. (1988), quienes afirmaron que la forma intermedia exhibe pocas semillas por vaina $(4.2 \mathrm{vs}$. 5.8) y tienen semillas más grandes (11.9 vs. $6.3 \mathrm{~g}$ en 100 semillas) que las formas silvestres. Se puede inferir entonces que las diferencias entre estos dos grupos son menores y que la forma intermedia o tipo maleza, es más cercana a la forma silvestre.

Al interior de los grupos también hubo diferencias significativas entre poblaciones $(\mathrm{P} \leq 0.05)$ en las formas intermedia y silvestre, en unos pocos caracteres (Cuadro 3). En longitud de vaina la población intermedia ROL 227-3 mostró el valor mayor $(10.8 \mathrm{~cm})$ y ROL $168 \mathrm{el}$ menor $(8.14 \mathrm{~cm})$. En la forma silvestre se observaron diferencias para longitud y área foliar del foliolo central; en ambos casos, ROL 242 presentó los foliolos más pequeños. Estos resultados muestran la escasa variabilidad fenotípica y genética existente entre las poblaciones incluidas dentro de los grupos.

El análisis de agrupamiento mediante la distancia euclidiana (Figura 1), muestra la separación de las tres formas de frijol. Las poblaciones domesticadas forman un grupo que se separa de las silvestres a una distancia de 2.08, mientras que las silvestres se diferencian de las intermedias a una distancia de 1.23; es decir, las formas silvestre e intermedia son más cercanas entre sí que con respecto a los frijoles cultivados. Estos resultados presentan similitud con lo encontrado por Zizumbo et al. (2005), al separar las formas cultivadas, intermedias y silvestres de frijol común. 

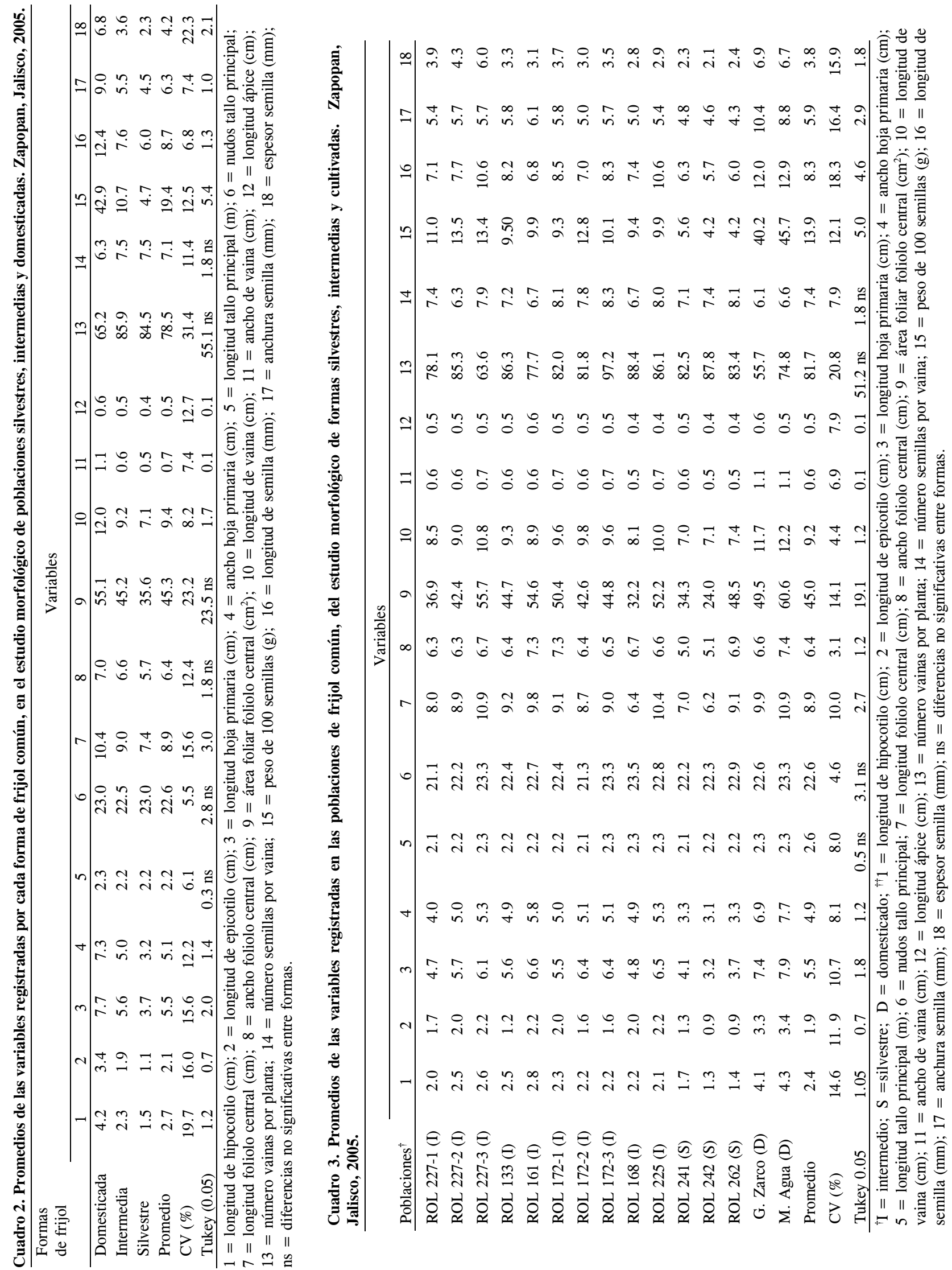


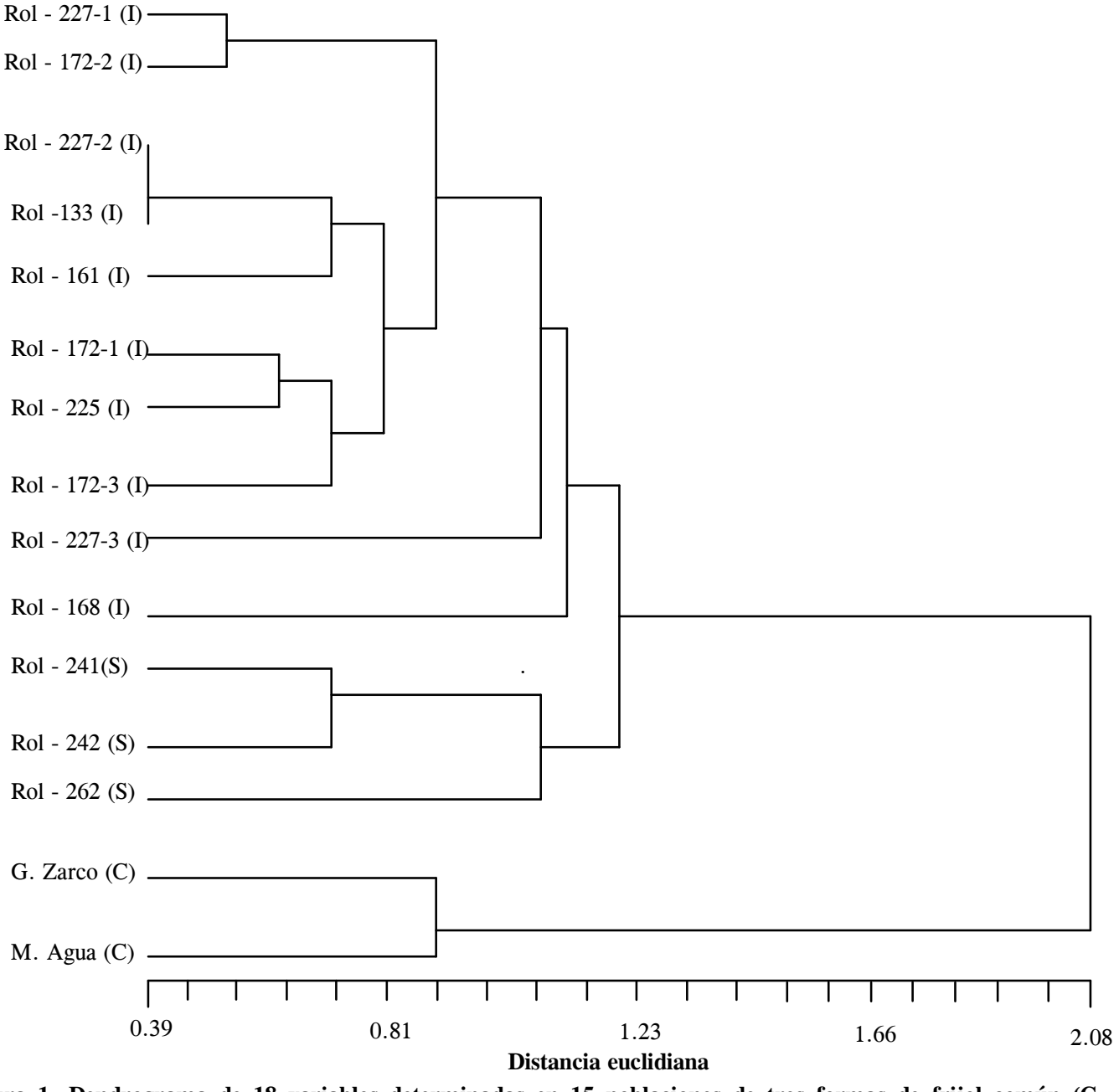

Figura 1. Dendrograma de 18 variables determinadas en 15 poblaciones de tres formas de frijol común $(C=$ cultivado; $\mathbf{S}$ = silvestre; $\mathbf{I}=$ intermedio).

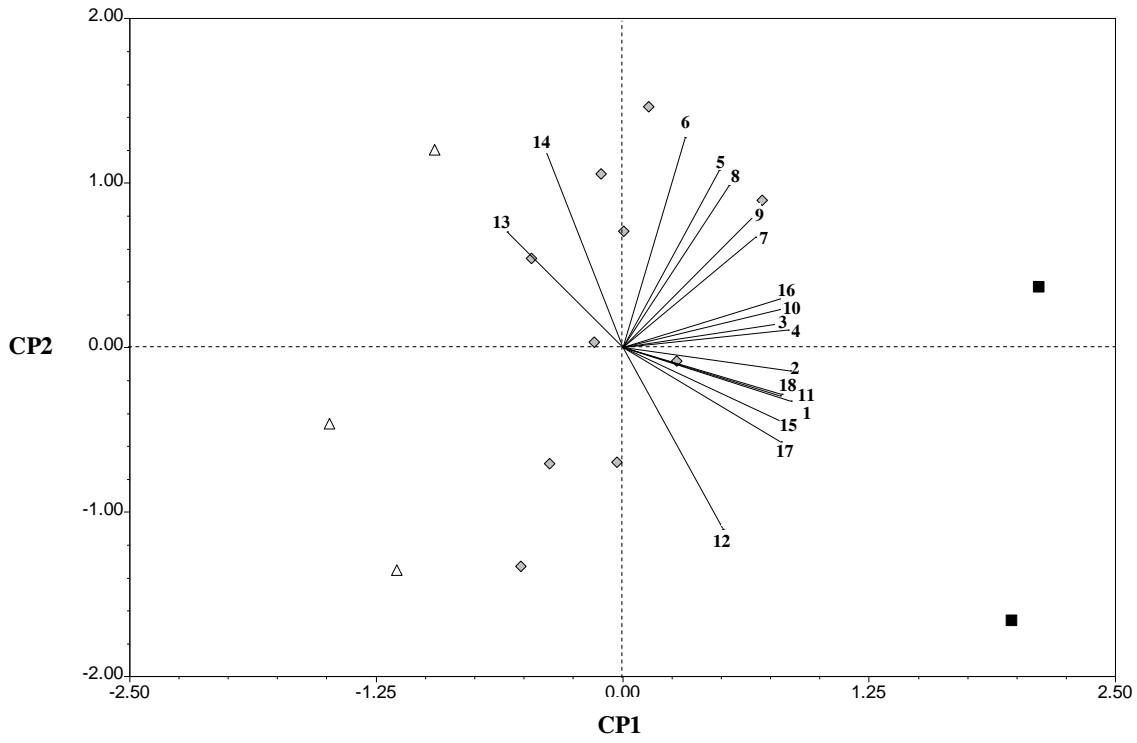

Figura 2. Ubicación de las unidades taxonómicas (poblaciones) y comportamiento de los vectoresvariable en base al análisis de componentes principales (CP) de 18 variables cuantitativas de frijol común ( $\diamond$, intermedios; $\Delta$, silvestres; $\sqcap$, cultivados). 
En el análisis "Biplot" (Figura 2) se encontró que los dos primeros componentes principales (CP) explicaron $77.6 \%$ de la suma de cuadrados de las variables originales, donde el primer componente aportó $64.3 \%$ de ese total. El CP1 permitió separar con claridad las tres formas de frijol trepador. Los frijoles domesticados se localizan en el extremo derecho del lado positivo, las poblaciones silvestres en el extremo izquierdo del lado negativo, y los intermedios en la parte media del gráfico. De las 10 formas intermedias incluidas, seis se encuentran del lado negativo y cuatro del lado positivo, en ambos casos cerca del valor central (0.00). Por su posición en la gráfica, las formas intermedias de frijol son más cercanas a las poblaciones silvestres, con excepción de ROL-227-3 que se acerca más a los frijoles domesticados, lo cual se debe principalmente a su mayor tamaño de vainas. Estos resultados son similares a los encontrados en el análisis de promedios (Cuadros 2 y 3) y en el dendrograma de la Figura 1.

Por la dirección y ángulo entre los vectores-variable que definen el grado de correlación, un grupo lo forman los caracteres número de vainas por planta (13) y número de semillas por vaina (14). Su proximidad espacial con las formas de frijol silvestre, revela su asociación con estas unidades taxonómicas. Un segundo grupo compacto de alta correlación, lo integran los vectores longitud de vaina (10), longitud de semilla (16), longitud de hoja primaria (3) y ancho de hoja primaria (4), de mayor valor en las formas domesticadas (Cuadro 2) y con proximidad espacial a las formas cultivadas (Figura 1). Un tercer grupo de comportamiento similar, lo constituyen los vectoresvariable de longitud de epicotilo (2), longitud de hipocotilo (1), anchura de vaina (11), espesor semilla (18), peso de semilla (15) y ancho de semilla (17), de alta correlación entre sí y con proximidad espacial hacia las formas domesticadas. Los vectores-variable longitud de ápice (12), nudos del tallo (6) y longitud del tallo (5), en general, no mostraron correlación con las demás variables y tampoco se asociaron con las unidades taxonómicas.

Es posible entonces afirmar que las poblaciones de frijol estudiadas constituyen tres grupos diferentes. La forma domesticada es diferente a la forma silvestre y exhibe diferencias notables en los caracteres de tamaño de fruto y semilla, caracteres del mayor interés humano durante el proceso de domesticación. Los frijoles tipo maleza forman un grupo intermedio, morfológicamente más cercanos a los silvestres. Los resultados también sugieren que el origen de las formas intermedias (tipo maleza) son producto de la hibridación natural entre poblaciones domesticadas y silvestres, y que en los sitios donde coexisten de manera simpátrica puede ocurrir polinización cruzada y flujo genético entre las dos formas principales de frijol común como proponen Delgado et al. (1988), Debouck et al. (1993) y Zizumbo et al. ( 2005). Dicho flujo genético representa un potencial para incrementar la variabilidad genética y las posibilidades de mejoramiento de la forma cultivada.

\section{CONCLUSIONES}

Las formas cultivada, silvestre e intermedia de frijol común de hábito trepador, constituyen tres grupos con características morfológicas diferentes. Los frijoles domesticados muestran mayores diferencias morfológicas en relación a los silvestres y los tipo maleza. Los frijoles tipo maleza forman un grupo intermedio, morfológicamente más cercano a las poblaciones silvestres. Los caracteres longitud y anchura de vaina, longitud, anchura y espesor del grano y peso de semilla entre otros, registraron mayores valores en las poblaciones domesticadas que en las silvestres, lo que permite suponer que dichos caracteres recibieron mayor atención durante el proceso de domesticación del frijol. Las variables longitud de tallo y número de entrenudos, no mostraron diferencias entre las formas de frijol trepador. Los caracteres número de vainas por planta y número de semillas por vaina, no se redujeron en la forma domesticada y se asocian con el mayor potencial de rendimiento de los frijoles cultivados.

\section{AGRADECIMIENTOS}

Al Sistema Nacional de Recursos Fitogenéticos (SINAREFI) coordinado por el Sistema Nacional de Inspección y Certificación de Semillas (SNICS), de la Secretaría de Agricultura, Ganadería, Desarrollo Rural, Pesca y Alimentación, por el financiamiento que hizo posible la realización del presente trabajo a través del Proyecto BI-FRI-02.

\section{BIBLIOGRAFÍA}

Adams M W (1967) Basis of yield component compensation in crop plants with special reference to the field bean, Phaseolus vulgaris. Crop Sci. 7:505-510

Chacon M I, B Pickersgill, D G Debouck (2005) Domestication patterns in common bean (Phaseolus vulgaris L.) and the origin of the Mesoamerican and Andean cultivated races. Theor. Appl. Genet. 110:432-444.

Debouck D, R Hidalgo (1985) Morfología de la planta de frijol común. In: Frijol: Investigación y Producción. M López, F Fernández, A van Schoonhoven (eds). CIAT, Cali, Colombia. pp:7-47.

Debouck D, O Toro, O M Paredes, W C Jonson, P Gepts (1993) Genetic diversity and ecological distribution of Phaseolus vulgaris (Fabaceae) in Northwestern South America. Econ. Bot. 47:408-423.

Delgado-Salinas A, A Bonet, P Gepts (1988) The wild relative of Phaseolus vulgaris in Middle America. In: Genetic Resources 
in Phaseolus beans. P Gepts (ed). Kluwer Academic Publishers. pp:163-184.

Gepts P, D Debouck (1991) Origin, domestication and evolution of the common bean (Phaseolus vulgaris L.). In: Common Beans. Research for Crop Improvement. A van Schoonhoven, O Voysest (eds). C.A.B. International. pp:7-53.

Gepts P, T C Osborn, K Rashka, F A Bliss (1986) Phaseolin-protein variability in wild forms and landraces of the common bean (Phaseolus vulgaris): evidence for multiple centers of domestication. Econ. Bot. 40:451-468.

Kaplan L, L N Kaplan (1988) Phaseolus in Archaeology. In: Genetic Resources of Phaseolus Beans. P Gepts (ed). Kluwer Academic Publishers. pp:125-142.

Koenig R, S P Singh, P Gepts (1990) Novel phaseolin types in wild and cultivated common bean (Phaseolus vulgaris, Fabaceae). Econ. Bot. 44:50-60.

Koinange E M K, S P Singh, P Gepts (1996) Genetic control of the domestication syndrome in common bean. Crop Sci. 36:10371045.

Kwak M, P Gepts (2009) Structure of genetic diversity in the two major gene pools of common bean (Phaseolus vulgaris L., Fabaceae). Theor. Appl. Genet. 118:979-992.

Kwak M, J A Kami, P Gepts (2009) The putative Mesoamerican domestication center of Phaseolus vulgaris is located en the Lerma-Santiago Basin of México. Crop Sci. 49:554-563.
Papa R, P Gepts (2003) Asymmetry of gene flow and differential geographical structure of molecular diversity in wild and domesticated common bean (Phaseolus vulgaris L.) from Mesoamerica. Theor. Appl. Genet. 106:239-250.

Poncet V, T R Aboubakry, P Gepts (2004) Quantitative trait locus analyses of the domestication syndrome and domestication process. Encyclopedia of Plant and Crop Science. Marcel Dekker, Inc. pp:1069-1073.

Rohlf F J (2000) Numerical Taxonomy and Multivariate Analysis System, NTSYSpc, version 2.1.

Sánchez G J J (1995) El análisis biplot en clasificación. Rev. Fitotec. Mex. 18:188-203.

Singh S P, P Gepts, D G Debouck (1991) Races of common bean (Phaseolus vulgaris, Fabaceae). Econ. Bot. 45:379-396

Toro O, J Tohme, D Debouck (1990) Wild bean (Phaseolus vulgaris L.): Description and distribution. International Board for Plant Genetic Resources (IBPGR) and Centro Internacional de Agricultura Tropical (CIAT). Cali, Colombia. $106 \mathrm{p}$.

White J, J Izquierdo (1989) FRIJOL: Fisiología del Potencial de Rendimiento y la Tolerancia al Estrés. CIAT-FAO. Santiago, Chile. $91 \mathrm{p}$.

Zizumbo-Villarreal D, P Colunga, E Payro, P Delgado-Valerio, P Gepts (2005) Population structure and evolution dynamics of wild-weedy-domesticated complexes of common bean in a Mesoamerican region. Crop Sci. 45:1073-1083. 